\title{
Humanized GHR106 Monoclonal Antibody is a Biosimilar GnRH Antagonist
}

\author{
Gregory Lee* \\ UBC Center for Reproductive Health, Canada
}

*Corresponding author: Gregory Lee, UBC Center for Reproductive Health, Vancouver, 9117 Shaughnessy Street, Vancouver, Canada, V6P 6R9, Canada

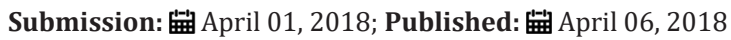

Keywords: Biosimilar GnRH antagonist; GnRH antagonist; Antide; Cetrorelix; Humanize; GHR106 monoclonal antibody; GHR106 of IgG4 isotype

\section{Opinion}

GHR106 is a monoclonal antibody generated against human GnRH receptor. The humanized forms of GHR106 exhibit almost identical biological properties to those of decapeptide $\mathrm{GnRH}$ antagonists such as Antide and Cetrorelix. The biosimilarity between these two GnRH receptor ligands was based on the studies of (1) binding affinity and specificity as well as immunohistochemical staining, (2) induced apoptosis to cancer cells and (3) effects of ligand- receptor bindings on gene regulations of cancer cells. Furthermore, the half-life of GHR106 antibody can be reduced from days to hours by means of antibody fragmentations to (Fab')2 or Fab. The results of these biosimilar studies suggest that humanized GHR106 with IgG4 isotype can be utilized clinically with biosimilar functions as antibody-based GnRH antagonist except, with higher molecular size and longer half-life as compared to those of peptide GnRH antagonists (days vs. hours).

GHR106 is a monoclonal antibody generated in mice against an oligopeptide corresponding to N1-29 amino acid residue in the extracellular domain of human GnRH receptor found in the anterior pituitary, cancer cells or reproduction-related normal organs or tissue in humans. The murine and humanized monoclonal antibody of GHR106 have been demonstrated to be bioequivalent in biological and immunological properties.

Numerous experiments have been performed to reveal high degree of biosimilarity between humanized GHR106 and GnRH decapeptide antagonist such as Antide and Cetrorelix. The mechanisms of actions of both GnRH peptide antagonist and GHR106 can behave biosimilarly, in several different cell types. Depending on the sites of ligand binding, the mechanisms of action may be dramatically different. For example, upon ligand interactions with the anterior pituitary, suppression of LH/FSH release can result in inhibition of ovulation. For bindings to other normal human tissues or cancer cells, apoptosis of these cell types can be induced. Based on these observations, humanized GHR106 should in principle serve as an alternative biosimilar antibody form of GnRH antagonist. Both can be utilized clinically for ovulation inhibition and other fertility- related indications. At the same time, long acting GHR106 in IgG1 subtype can be used for cancer treatment, similar to that of peptide antagonist. For example, in the case of interactions with the anterior pituitary, the main actions of GnRH peptide antagonist or GHR106 may be on the suppression of LH/FSH upon the ligand binding to GnRH receptor, which will result in ovulation inhibitions.

However, in the case of fertility treatment, minor structural modifications may be required in the IgG Fc regions to eliminate the undesirable effecter functions associated with antibody ligands of certain IgG isotypes (eg. IgG, IgG2, and IgG3). Therefore, GHR106 in IgG4 isotype will be employed for drug development to eliminate the possibility of complement-dependent cytotoxicity (CDC) and antibody-dependent cellular cytotoxicity (ADCC) reactions upon the ligand binding to the anterior pituitary.

In addition, the circulation half-life can be adjusted to optimize clinical conditions and requirements. This objective can also be achieved by fragmentations or size-reductions of the original GHR106 (IgG4) antibody into (Fab')2, Fab or ScFv. Based on these considerations, a series of antibody-based GnRH antagonists can be generated as drugs of different half-lives for clinical applications of many fertility-related indications.

Therefore, we believe that humanized GHR106 of IgG1 isotype with effective CDC and ADCC activities can be utilized clinically for cancer-treatments. However, when served as the biosimilar antifertility drugs, humanized GHR106 of IgG4 isotype should be an ideal choice, in which the effector functions are eliminated to avoid any complications. 
Creative Commons Attribution 4.0

International License

For possible submissions Click Here

Submit Article

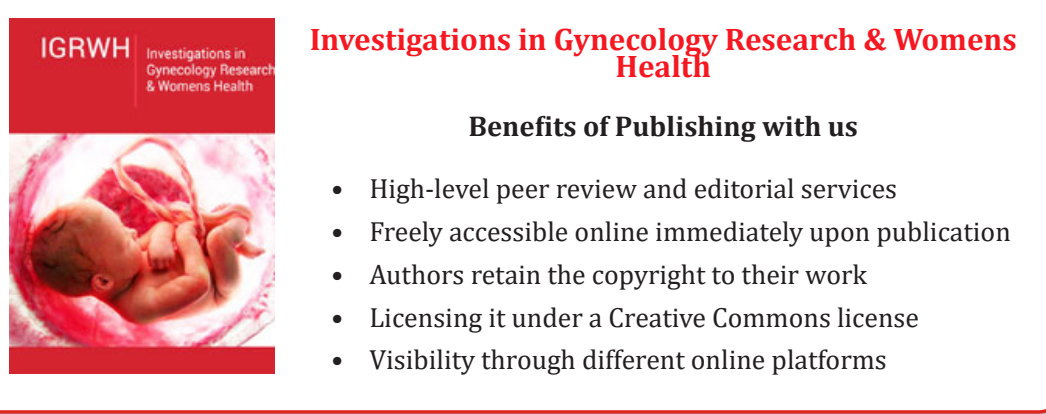

